\title{
An RFID System Based MCLT System with Improved Privacy
}

\author{
Jin Kwak ${ }^{1, \star}$, Keunwoo Rhee ${ }^{2}$, Namje Park ${ }^{2,3}$, Howon Kim ${ }^{3}$, Seungjoo Kim², \\ Kouichi Sakurai ${ }^{1}$, and Dongho Won ${ }^{2, \star \star}$ \\ ${ }^{1}$ Faculty of Information Science and Electrical Engineering, \\ Kyushu University, Japan \\ $\{j k w a k$, sakurai\}@itslab.csce.kyushu-u.ac.jp \\ ${ }^{2}$ Information Security Group, Sunkyunkwan University, Korea \\ \{kwrhee, skim, dhwon\}@security.re.kr \\ ${ }^{3}$ Information Security Research Division, ETRI, Korea \\ \{namejepark, khw\}@etri.re.kr
}

\begin{abstract}
Radio Frequency Identification (RFID) systems are increasingly becoming accepted for many EPC Network applications. However, RFID systems have some privacy problems. In this paper, a system for missing child location tracking in the EPC Network applications, is proposed. The proposed system improves security and privacy compared to existing applications, while also keeping in line with traditional procedures, commonly accepted by most industrial applications. The proposed MCLT (Missing Child Location Tracking) system can protect users' privacy while providing location tracking of the RFID tag.
\end{abstract}

Keywords: EPC Network, RFID system, application service, privacy, security, location tracking.

\section{Introduction}

The main technology of the EPC Network consists of a RFID system that recognizes and manages RFID tags by use of the Radio Frequency (RF) signal. Low-cost RFID tags can be read, and information can be updated without any physical contact. Therefore, RFID systems have become popular for automated identification in EPC Network applications [8,14,20,23,25]. However, this technology creates new problems, the most important being the invasion of users' privacy. Thus, several methods for protecting the users' location privacy have been proposed $[11,12,17,19,21,22,28,29]$.

However, previous protocols do not resolve security and privacy problems such as location tracking, location history disclosure, and counterfeiting (see [19] for

\footnotetext{
* The first author was supported by the Korea Research Foundation Grant funded by the Korean Government (MOEHRD). (KRF-2006-214-D00152).

** Corresponding Author: Dongho Won (dhwon@security.re.kr), He was supported by the University IT Research Center Project funded by the Korean Ministry of Information and Communication.
} 
more details). In addition, the proposed protocols are not suitable for ubiquitous computing environment, and using distributed databases. In particular, although the RFID tag enables location based applications to be more effective, it may also allow access to information regarding users location, without their agreement. Location based services rely on the availability of user location information. However, location information is sensitive, therefore, releasing this information to random entities may create security and privacy problems. In particular, the previous protocols are not suitable for location based systems for missing child location tracking in EPC Network applications.

In this paper, a system for missing child location tracking using EPC Network applications is proposed. In this proposed MCLT system: (1) the user registers with the registration authority ( $R M A$ in this paper), (2) the registration authority issues the user with a tag which, for privacy, includes an anonymous-EPC, (3) access to tag information is permitted only by authorized administrators, and (4) for anonymity, the authentication process is performed using a random response at each authentication process between the tag and the reader. In addition, the location of a user can be securely and effectively tracked by authorized administrators, such as in the case of finding a missing child (the tagged user (tag holder)).

The subsequent sections of this paper are organized as follows. After shortly discussing the EPC Network and the associated components in Section 2, security and privacy requirements for the proposed system are presented in Section 3. In Section 4, the EPC Network based MCLT (Missing Child Location Tracking) System is proposed, enabling the protection of users' privacy, while allowing location tracking of the tagged user (tag holder) by authorized administrators when a child is missing. Finally, conclusions are presented in Section 5.

\section{The EPC Network}

The EPC Network includes several components, the EPC, tag, reader, EPC Information Server (EPCIS), Object Name Service (ONS), and Middleware $[1,2,5,6,7,10,16,24]$.

-EPC: The binary representation of the EPC (Electronic Product Code), a combination of Header, EPC Manager, Object Class, and Serial Number. Header identifies the version, length, tag type, and structure of the code. EPC Manager identifies a company, a manager, or an organization. In short, it indicates a manufacturer ID. Object Class indicates article classification (manufacturer's product ID). The class number must be unique for all given domains. Serial Number is unique for every class and non-repeating for each object class code.

-EPC Middleware: EPC Middleware manages EPC data received from the reader, provides alerts, and reads information for communication to the EPCIS (EPC Information Services) or the company's other existing information systems. EPCglobal is developing a software interface standard for services 
enabling data exchange between an EPC reader or network of readers and information systems. EPC middleware designed to process the stream of tag data coming from one or more readers, and this particular piece of software manages readers.

-RFID Tags and Readers: RFID systems are basically composed of tags and readers. The tag generally consists of an IC chip and an antenna. The IC chip in the tag is used for data storage and logical operations, whereas the coiled antenna is used for communication with the reader. The reader generally consists of an RF module, control unit, and coupling element to interrogate electronic tags via $R F$ communication. The RFID tag is either an active or a passive tag ${ }^{1}$. The EPC is stored on this tag. Tags communicate their EPCs to readers using a $\mathrm{RF}$ signal. The readers communicate with tags using the RF signal and deliver information to application systems with EPC middleware.

-EPC Information Service: EPCIS (EPC Information Services) enables users to exchange EPC-related data with trading partners through the EPC Network. EPCIS provides EPC Network related data available in PML format to request services. Data available through the EPCIS may include tag data collected from EPC middleware such as date of manufacture, expiry date, and product information.

-Discovery Service: The ONS provides a global lookup service, translating EPCs into one or more Internet Uniform Reference Locators (URLs), where further information regarding the object may be retrieved. In short, the ONS provides yellow page services for the EPC Network, allowing participants to quickly discover the server in the EPC Network containing the information associated with a particular EPC. The ONS works same as Domain Name Service (DNS), the foundation naming protocol for the Internet.

\section{Security and Privacy Requirements for the Proposed System}

In EPC Network applications, especially location based services such as missing child location tracking services, the availability of the users' location information is depended on. However, users usually prefer their location information to be kept secret, because their personal location information is regarded as sensitive. Therefore, only authorized entities should have access to users' location information, and only when necessary [15].

In this section, security and privacy requirements for the proposed system are described. For users' privacy, the EPC of the tag should not be known to the reader or system; only authentication of the tag should be provided. In addition, only authorized administrators should have access to location information, and only when necessary [15].

${ }^{1}$ The active $\operatorname{tag}$ possesses a battery and actively transmits information to the reader for communication. The passive tag must be inductively powered from the RF signal of the reader since RFID tags usually do not possess their own battery power supply. In this paper, the passive tag used to accomplish a hash operation. 
Anonymity: The recipient of a response generated by the tag can verify that it is a valid response of the query, but cannot discover which tag created it.

Unlinkability: The recipient of a response generated by the tag can verify that it is a valid response of the query, but cannot be decided whether two responses have been generated by the same tag.

Traceability: The EPC of the tag should be encrypted and stored in a Registration and Management Authority $(R M A)$. Location tracking of the tag holder should be possible only through the cooperation of authorized administrators, and only in an emergency situation, such as when a child goes missing.

\section{Proposed MCLT System}

For the proposed system, the Registration and Management Authority (RMA) is defined. The $R M A$ is a trusted security center managed by a public institution for the registration and location tracking of a user. The RMA consists of a Registration Server and EPC Network components.

The Registration Server issues a tag to the user and stores related information such as telephone number and address of the user in the database. To satisfy security and privacy requirements (see section 3.), the Registration Server generates the $E I D_{i}$ (encrypted value of the $\mathrm{EPC}_{i}$ ) of the $\mathrm{Tag}_{i}$. In this paper, for encryption of the $E I D_{i}$, cryptographic secret sharing methods are adopted $[3,4,9,13,18,26,27]$.

\begin{tabular}{|c|c|c|c|c|}
\hline EPC : & Header & EPC Manager & Object Class (OC) & Serial Number (SN) \\
\hline Proposed EID: & Header & $\begin{array}{l}\text { EID Manager } \\
\text { (RMA's ID) }\end{array}$ & & pted value \\
\hline
\end{tabular}

Fig. 1. EID : encrypted value of the EPC

Fig. 1 present EID and Fig. 2 presents the proposed system. For the proposed system example, it is assumed that the user travels around three local EPC Network zones in a regular sequence (e.g., school, wear shop, and restaurant zone).

\subsection{User Registration}

Fig. 3 presents the user registration process for the proposed system.

\section{[ Notations and Parameters ]}

- $P$ : a set of participant $A d m_{i} \mathrm{~s}, P=\left\{A d m_{1}, A d m_{2}, \cdots, A d m_{n}\right\}$

$-p:$ a large prime number, where $p>2^{512}$

$-q$ : a prime number, where $q \mid p-1$

- $g:$ an element over $\mathbb{Z}_{p}$, where $\operatorname{ord}(g)=q$

- $A d m_{i}(1 \leq i \leq n)$ : administrators of $R M A$. 


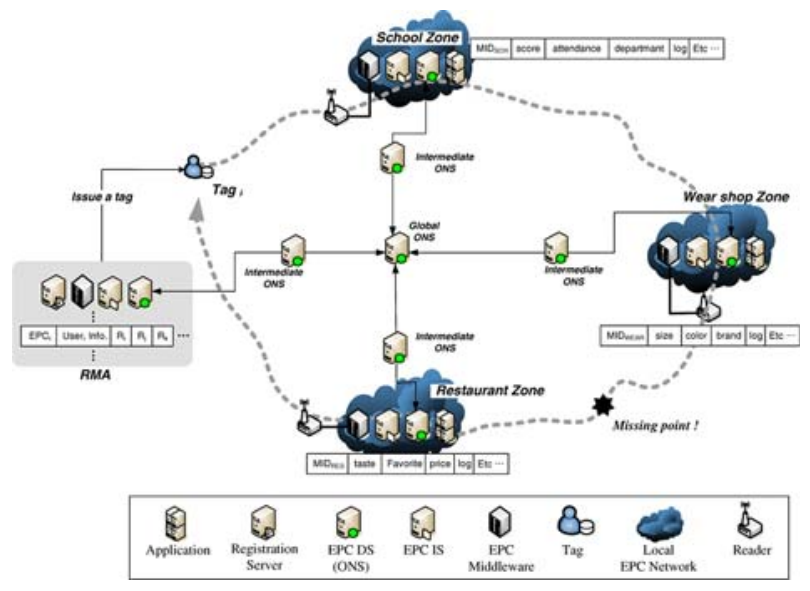

Fig. 2. The EPC Network based MCLT System with Improved Privacy

$-y_{A d m}:$ a group public key of administrators

- ENC() : a public key encryption scheme

- $H()$ : cryptographically secure hash-functions

- EPC $C_{i}$ : the EPC of the $\operatorname{tag}_{i}$

- $E I D_{i}$ : encrypted value of $E P C_{i}\left(E I D_{i}=E N C\left(E P C_{i}\right)\right)$

- MID $D_{i}$ : meta-ID of $\operatorname{Tag}_{i}$

- $I D_{i}:$ ID of the local EPC Network

$-R_{R}, R_{i}$ : random number chosen by the reader and the $\operatorname{tag}_{i}$

\section{[ User Registration ]}

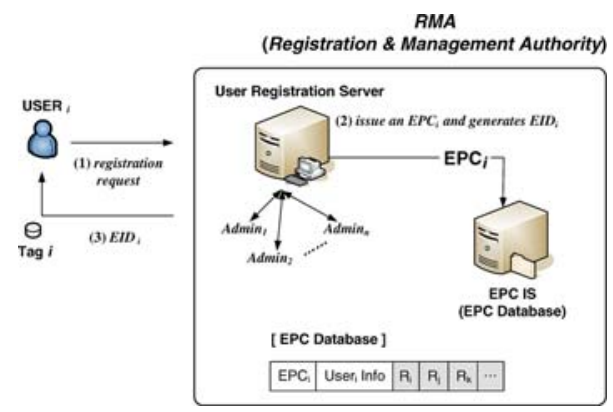

Fig. 3. User Registration for the proposed system

1. $\mathrm{User}_{i}$ make an application ${ }^{2}$ for registration to $R M A$.

2. Registration server in $R M A$,

(a) issues an $\mathrm{EPC}_{i}$ and then subsequently encrypts it.

(b) the encrypted value $E I D_{i}$ is written to the $\operatorname{Tag}_{i}$.

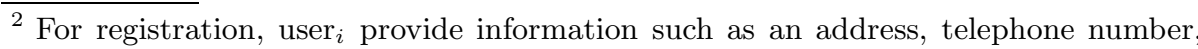
family exemplification, and so on. The RMA stores this information in a database. 


\section{(The generation of the Group public key)}

(1) Every administrator $\left(A d m_{i} \mid i \in\{1, \cdots, n\}\right)$ selects $r_{i} \in_{R} Z_{q}$ at random and broadcasts $y_{i}=g^{r_{i}} \bmod p$ to all other administrators in the set $S_{i}$.

$$
S_{i}=\left\{A d m_{j} \mid j \in\{1, \cdots, n\} \quad \text { AND } i \neq j\right\}
$$

(2) To distribute $r_{i}$, each $A d m_{i}$ randomly selects a polynomial $f_{i}$ of degree $t-1$ in $Z_{q}$ such that $f_{i}(0)=r_{i}$, i.e.,

$$
f_{i}(x)=r_{i}+a_{i, 1} x+a_{i, 2} x^{2}+\cdots+a_{i, t-1} x^{t-1}
$$

with $a_{i, 1}, \cdots, a_{i, t-1} \in_{R} Z_{q}$, and transmits $f_{i}(j) \bmod q$ to $A d m_{j}$ in a secure manner $(\forall j \neq i)$. Each $A d m_{i}$ also broadcasts values

$$
g^{a_{i, 1}} \bmod p, \cdots, g^{a_{i, t-1}} \bmod p
$$

(3) From distributed $f_{j}(i)(\forall j \neq i), A d m_{i}$ checks whether, for each $j$,

$$
g^{f_{j}(i)}=y_{j} \cdot\left(g^{a_{j, 1}}\right)^{i^{1}} \cdots\left(g^{a_{j, t-1}}\right)^{i^{t-1}} \bmod p
$$

(4) Let $H=\left\{A d m_{j} \mid A d m_{j}\right.$ is not detected to be cheating at step 3$\}$. Every $A d m_{i}$ computes the share $s_{i}$ secretly, and computes the group public key $y_{A d m}$.

$$
s_{i}=\sum_{j \in H} f_{j}(i), \quad y_{A d m}=\prod_{j \in H} y_{j}
$$

\section{(The encryption of $E P C$ )}

(5) To encrypt each $\mathrm{EPC}_{i}, A d m_{1}$ picks $t_{i, 1} \in_{R} Z_{q}$ and computes $g^{t_{i, 1}} \bmod p$. Then, $A d m_{1}$ transmits the result to $A d m_{2}$.

(6) The $A d m_{2}$ selects $t_{i, 2} \in_{R} Z_{q}$ and computes $\left(g^{t_{i, 1}}\right)^{t_{i, 2}} \bmod p$. Then, $A d m_{2}$ transmits the result to $A d m_{3}$.

(7) The final participant $A d m_{i}(i \neq n)$ computes $g^{t_{i}}=\left(g^{t_{i, 1}, t_{i, 2}, \cdots}\right)^{t_{i, n}} \bmod p$ and broadcasts the result to all other $A d m_{n}$.

(8) Through the cooperation of $\mathrm{n} A d m_{i}$, the ciphertext of $\mathrm{EPC}_{i}$ is generated as follows. Where $\mathrm{OC} \| \mathrm{SN}_{i}$ is the value of concatenated object class and serial number, detailed in Fig. 1.

$E I D_{i}=E N C\left(E P C_{i}\right)=\left(g^{t_{i}}, A\right)$, where $A=\left(\left(y_{A d m}\right)^{t_{i}} \cdot\left(O C \| S N_{i}\right)\right.$ $\bmod p)$

3. The $\operatorname{Tag}_{i}$ is issued to user $_{i}$. The $\operatorname{Tag}_{i}$ contains the encrypted value EID instead of the $\mathrm{EPC}_{i}$.

\subsection{MID Registration and Tag Authentication}

The following steps demonstrate the MID registration and authentication process in each local EPC Network. In this subsection, the registration and authentication processes in the school zone (local EPC Network) are described. The processes of other local EPC Network zones are identical to the processes in the school zone. Fig. 4 demonstrates MID registration and tag authentication in the case of the school zone.

\section{[ MID Registration ]}

The following steps represent the MID registration process in each local Network, through the EPC Network system [7,16]. The detailed protocol of MID registration in the school zone as follows: 


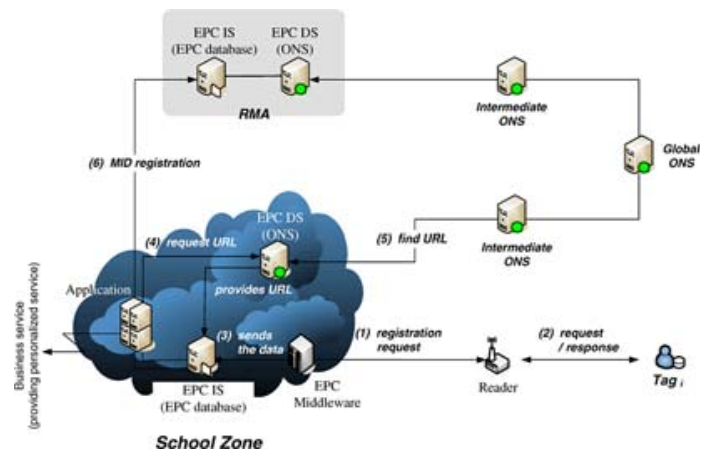

Fig. 4. MID Registration and Tag authentication processes in the School zone

1. The school zone EPC Network system transmits the registration request to the $\operatorname{Tag}_{i}$.

2. $\operatorname{Tag}_{i}$ generates a random number $R_{i}$, and then computes $M I D_{S C H}$ and stores it. Then the $\operatorname{Tag}_{i}$ transmits $M I D_{S C H}$ to the system. Fig. 5 presents the updated information in the $\mathrm{Tag}_{i}$.

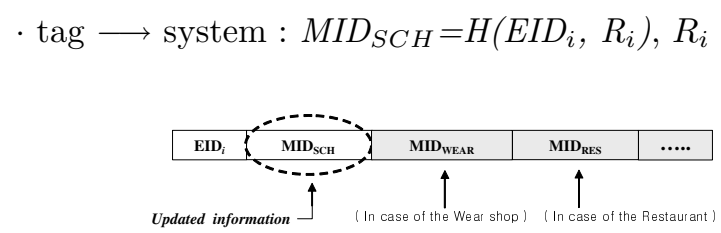

Fig. 5. Updated information in the $\operatorname{Tag}_{i}$

3. When the local middleware receives data from the $\mathrm{Tag}_{i}$, the local middleware can be configured to transmit the data further to the local EPCIS. Then local EPCIS stores $M I D_{S C H}$ in a database for authentication of the $\operatorname{Tag}_{i}$.

4. After Step 3, the local EPCIS searches the URL of the $\operatorname{Tag}_{i}$ 's issuer for $M I D_{S C H}$ registration. In the case where the URL of the $\operatorname{Tag}_{i}$ is unknown to the system, the system will consult the ONS to obtain the URL of the $\operatorname{Tag}_{i}$.

5. If the local ONS is unable to transform the $\mathrm{Tag}_{i}$ into an URL, the ONS will query other ONS systems higher in the ONS hierarchy, and may potentially make an enquiry to the global ONS via the Internet. The correct URL will then be transmitted to the local EPCIS.

6. Then, local EPCIS transmits the registration message and $R_{i}$ to the $R M A$. The $R M A$ performs a brute-force search to retrieve the EPC; Upon receiving the $R_{i}$, the $R M A$ calculates $M I D_{S C H}$ for all EPC's stored in the database. Only when it finds a match to $M I D_{S C H}$ (received from the system) has it identified the right EPC. If the above procedure is successful, the $R M A$ stores 


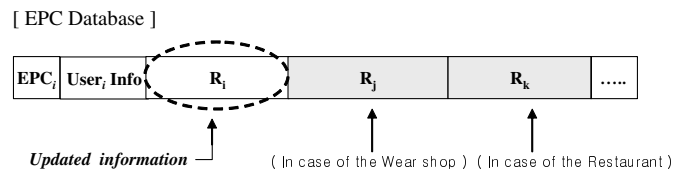

Fig. 6. Updated information of the $\mathrm{Tag}_{i}$ in $R M A$

$R_{i}$ in their database (see fig. 6-demonstrates how information is updated in the $R M A$ for the $\operatorname{Tag}_{i}$.).

\section{[ Tag authentication ]}

After completing MID registration, the local EPC Network performs authentication of the tag $[15,19]$.

Fig. 7 presents the detailed protocol of authentication in the school zone.

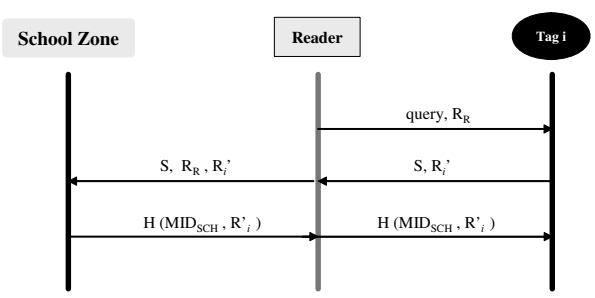

Fig. 7. Tag authentication protocol

(1) RFID reader transmits a query to the $\operatorname{Tag}_{i}$ with $R_{R}$.

(2) The $\operatorname{Tag}_{i}$ generates random number $R_{i}^{\prime}$. Then the $\operatorname{Tag}_{i}$ computes a response $S\left(=H\left(M I D_{S C H}, R_{i}^{\prime}, R_{R}\right)\right)$ and transmits it to the reader with $R_{i}^{\prime}$.

(3) The reader transmits $R_{R}$ to the system with $S$ and $R_{i}^{\prime}$.

(4) The local EPC Network system computes hash value $S^{\prime}$ using the stored $M I D_{S C H}$. Then the system subsequently compares it with $S$, received from the reader to authenticate the $\operatorname{Tag}_{i}$.

$$
\text { - System : received } S \stackrel{?}{=} \quad \text { computed } S^{\prime}
$$

If the authentication is successful, the system transmits $H\left(M I D_{S C H}, R_{i}^{\prime}\right)$ to the reader.

(5) The reader transmits the $H\left(M I D_{S C H}, R_{i}^{\prime}\right)$ received from the system to the $\operatorname{Tag}_{i}$.

Then, to authenticate the system, the the $\operatorname{Tag}_{i}$ computes $H\left(M I D_{S C H}, R_{i}^{\prime}\right)$ and compares it with the value received from the reader. If authentication is successful, the system is authenticated.

$$
\cdot \operatorname{tag} \text { : computed } H\left(M I D_{S C H}, R_{i}^{\prime}\right) \stackrel{?}{=} \text { received } H\left(M I D_{S C H}, R_{i}^{\prime}\right)
$$




\subsection{Location Tracking Process}

In this subsection, the location tracking process in the proposed system in case of finding a missing child, is described. Fig. 8 presents the processes of location tracking the $\operatorname{Tag}_{i}$.

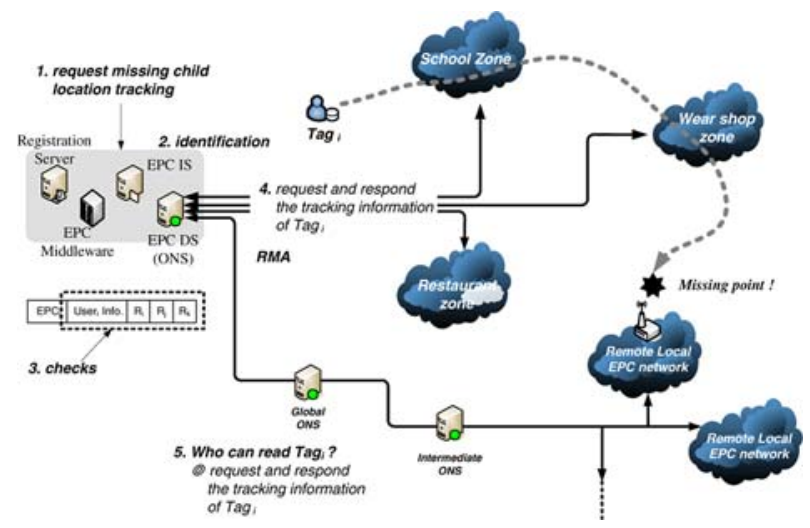

Fig. 8. Missing child location tracking

1. When parents are missing a child, an occurrence of a missing child is reported and location tracking of the tag is requested to the $R M A$. The parents transmit related information (e.g., such as address, telephone number, and family exemplification for verify the identity of the requestor) with a request message.

2. The $R M A$ verifies the identity of the requestor using the received information and the stored information in the user registration phase.

3. The $R M A$ searches the local EPC Network related specific to the $\mathrm{Tag}_{i}$. To achieve this step, the $R M A$ requires at least $t(n \geq t)$ shared information among $n$. Since the $M I D_{i}$ is encrypted. Therefore, only authorized administrators can identify the $M I D_{i}$ of the $\operatorname{tag}_{i}$. If $X=\left\{A d m_{1}, A d m_{2}, \cdots, A d m_{t}\right\}$ is a qualified subset to recover the $M I D_{i}$, the recovery phase is operated as follows;

(1) Through the cooperation of every $A d m_{i} \in X$, the value $r_{1}+r_{2}+\cdots+r_{t}$ $\bmod p$ is recovered using polynomial interpolation [26].

(2) Each $A d m_{i} \in X$ computes $\left(g^{t_{i}}\right)^{r_{1}}+r_{2}+\cdots+r_{t}$ using a stored $g^{t_{i}}$.

(3) Each $A d m_{i} \in X$ computes the $E I D_{i}$ as follows;

$$
E I D_{i}=A /\left(g^{t_{i}}\right)^{r_{1}}+r_{2}+\cdots+r_{t}
$$

4. To retrieve the specific local EPC Network for the $\operatorname{Tag}_{i}$, the $R M A$ computes the meta-ID $\left(M I D_{i}\right.$ of the $\left.\operatorname{Tag}_{i}\right)$.

$$
\begin{array}{clll}
\text { computes } & H\left(E I D_{i}, R_{i}\right) & \longrightarrow & M I D_{S C H} \\
\text { computes } & H\left(E I D_{i}, R_{j}\right) & \longrightarrow & M I D_{W E A R} \\
\text { computes } & H\left(E I D_{i}, R_{k}\right) & \longrightarrow & M I D_{R E S}
\end{array}
$$


5. Then, the $R M A$ requests the log information of the $\operatorname{Tag}_{i}$ and reads the $\operatorname{Tag}_{i}$ at each local EPC Network. Then the local EPC Network responds to the $\mathrm{Tag}_{i}$ read with a success or failure. If each local EPC Network fails to read the $\mathrm{Tag}_{i}$, the local EPC Network transmits the log information of the $\mathrm{Tag}_{i}$ to the $R M A$. (see fig. 9)

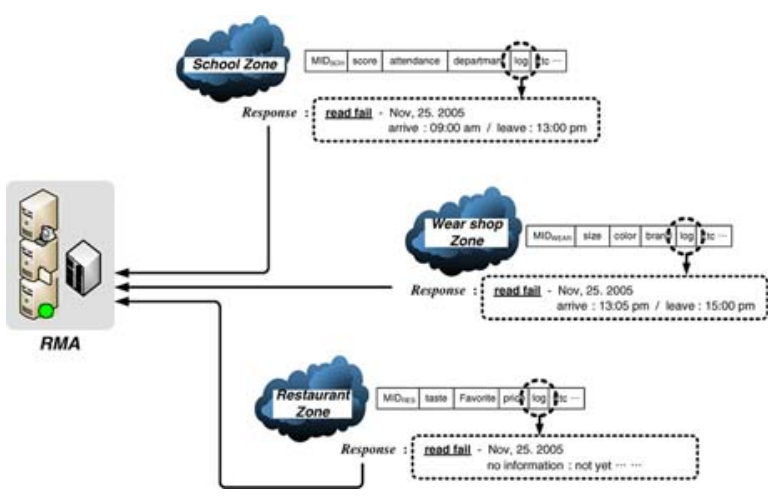

Fig. 9. Response of each local EPC Network

6. After receiving responses, the $R M A$ analyzes the received information (see in Fig. 9) and predicts where the child located, in the example below, this is between the wear shop and the restaurant.

7. The $R M A$ requests a remote local EPC Network (the local EPC Network of the wear shop, restaurant, and its outskirts) to read the $\mathrm{Tag}_{i}$ via the hierarchical ONS system. If the process does not find a missing child, the $R M A$ requests an extension to neighboring districts.

8. Finally, the $R M A$ retrieves the location of the missing child successfully. As a result, the missing child is now found.

\section{Conclusion}

In this paper, a system for missing child location tracking in EPC Networks, with improved privacy, is proposed. In the proposed system, the tag embeds $E I D$ instead of the EPC, to provide improved privacy for the user. Then registration authority issues tag to the user, which, for privacy, includes an anonymous-EPC.

In conclusion, the following four points are stated: (1) access to the information of the tag is permitted only to authorized administrators, (2) the authentication process is performed using a random response at each authentication process between the tag and the reader, and (3) the location of the tag user can be securely and effectively tracked by authorized administrators, such as, in the case of retrieving a missing child. (4) In addition, although the authorized administrator kept one tag, other tags do not add any security and privacy 
problems. Therefore, the proposed system can be satisfied with unlinkability, anonymity and traceability.

Acknowledgement. The 6th author, Kouichi Sakurai, is partially supported by Strategic International Cooperative Program, Japan Science and Technology Agency (JST).

\section{References}

1. D. L. Brock. The electronic product code (EPC): A naming scheme for objects. Technical Report MIT-AUTOID-WH-002, MIT Auto ID Center, 2001. Available from http://www.autoidcenter.org.

2. D. L. Brock. EPC Tag Data Specification. Technical Report MIT-AUTOID-WH025, MIT Auto ID Center, 2003. Available from http://www.autoidcenter.org.

3. C. Cachin. On-Line Secret Sharing. Cryptography and Coding: The 5th IMA Conference, LNCS 1025, pp. 190-198, Springer-Verlag, 1995.

4. L. Chen, D. Gollmann, C. J. Mitchell and P. Wild. Secret sharing with Reusable Polynomial. Australian Conference on Information Security and Privacy, ACISP 97, LNCS 1270, pp. 183-193, Springer-Verlag, 1997.

5. D. Engels. The Reader Collision Problem. Technical Report. MIT-AUTOID-WH007, MIT Auto ID Center, 2001. Available from http://www.autoidcenter.org.

6. D. Engels. EPC-256 : The 256-bit Electronic Product Code Representation. Technical Report MIT-AUTOID-WH-010, MIT Auto ID Center, 2003. Available from http://www.autoidcenter.org.

7. EPCglobal. The EPCglobal Network: Overview of Design, Benefits, and Security. 24 September 2004. Available from http://www.epcglobalinc.org.

8. D. M. Ewatt and M. Hayes. Gillette razors get new edge: RFID tags. Information Week, 13 January 2003. Available from http://www.informationweek.com.

9. P. Fedlman. A Practical scheme for Non-interactive Verifiable secret sharing. The 28th Annual Symposium on the Foundation of Computer Science, pp. 427-437, 1987.

10. K. Finkenzeller. RFID Handbook, John Wiley and Sons. 1999.

11. A. Juels and R. Pappu. Squealing Euros: Privacy protection in RFID-enabled banknotes. Financial Cryptography 2003, FC'05, LNCS 2742, pp. 103-121, SpringerVerlag, 2003.

12. A. Juels, R. L. Rivest and M. Szydlo. The Blocker Tag : Selective Blocking of RFID Tags for Consumer Privacy. 10th ACM Conference on Computer and Communications Security, CCS 2003, pp. 103-111, 2003.

13. S. Kim, S. Park and D. Won. Proxy Signatures, Revisited. International Conference on Information and Communications Security, ICICS'97, LNCS 1334, pp. 223-232, Springer-Verlag, 1997.

14. H. Knospe and H. Pobl. RFID Security. Information Security Technical Report, vol. 9, issue 4, pp. 39-50, Elsevier, 2004.

15. J. Kwak, K. Rhee, S. Oh, S. Kim, and D. Won. RFID System wuth Fairness within the frmaework of Security and Privacy. 2nd European Workshop on Security and Privacy in Ad hoc and Sensor Networks, ESAS 2005, LNCS 3813, Springer-Verlag, 2005.

16. K. S. Leong and and M. L. Ng. A Simple EPC Enterprise Model. Auto-ID Labs Workshop Zurich. 2004. Available from http://www.m-lab.ch 
17. M. Ohkubo, K. Suzuki, and S. Kinoshita. A Cryptographic Approach to "PrivacyFriendly" tag. RFID Privacy Workshop, Nov 2003. http://www.rfidprivacy.org/

18. T. P. Pedersen. A Threshold cryptosystem without a trusted party. Eurocrypt '91, LNCS 547, pp. 522-526, Springer-verlag, 1991.

19. K. Rhee, J. Kwak, S. Kim, and D. Won. Challenge-Response Based RFID Authentication Protocol for Distributed Database Environment. Second International Conference on Security in Pervasive Computing, SPC 2005, LNCS 3450, pp. 70-84, Springer-Verlag, 2005.

20. S. E. Sarma. Towards the five-cent tag. Technical Report MIT-AUTOID-WH-006, MIT Auto ID Center, 2001. Available from http://www.autoidcenter.org.

21. S. E. Sarma, S. A. Weis, and D. W. Engels. RFID systems, security and privacy implications. Technical Report MIT-AUTOID-WH-014, AutoID Center, MIT, 2002.

22. S. E. Sarma, S. A. Weis, and D. W. Engels. Radio-frequency identification systems. Workshop on Cryptographic Hardware and Embedded Systems, CHES02, LNCS 2523, pp. 454-469, Springer-Verlag, 2002.

23. S. E. Sarma, S. A. Weis, and D. W. Engels. Radio-frequency-identification security risks and challenges. CryptoBytes, 6(1), 2003.

24. T. Scharfeld. An Analysis of the Fundamental Constraints on Low Cost Passive Radio-Frequency Identification System Design. MS Thesis, Department of Mechanical Engineering, Massachusetts Institute of Technology, Cambridge, MA 02139, 2001.

25. Security technology: Where's the smart money? The Economist, pp. 69-70, 9 February 2002.

26. A. Shamir, How to share a secret. Communication of the ACM, vol. 21, pp. 120-126, 1979 .

27. M. Tompa and H. Woll. How to share a secret with cheater. Journal of Cryptology, vol. 1, pp. 133-138, 1988.

28. S. A. Weis. Radio-frequency identification security and privacy. Master's thesis, M.I.T. May 2003.

29. S. A. Weis, S. Sarma, R. Rivest, and D. Engels. Security and privacy aspects of low-cost radio frequency identification systems. First International Conference on Security in Pervasive Computing, SPC 2004, LNCS 2802, pp. 201-212, SpringerVerlag, 2004. 